\title{
Studies on $\mathrm{Li}$-doped $\mathrm{Ca}_{0.85} \mathrm{Sr}_{0.15} \mathrm{CuO}_{2}$; the prototype of cuprate family of oxide superconductors
}

\author{
B LAKSHMI BRINDA, U V VARADARAJU and G V SUBBA \\ RAO \\ Materials Science Research Centre, Indian Institute of Technology, Madras 600036, India
}

\begin{abstract}
The layer-type compound, $\mathrm{Ca}_{0.85} \mathrm{Sr}_{0 \cdot 15} \mathrm{CuO}_{2}$, which is the prototype of the cuprate family of high $T_{c}$ oxide superconductors is an electronic insulator, and has a low ordered moment at $300 \mathrm{~K}$ indicating that $\mathrm{Cu}^{2+}$ ions are highly correlated. Doping with lithium (up to $x=0.15$ ) decreases the room temperature resistivity drastically (from $10^{3} \mathrm{ohm} \cdot \mathrm{cm}$ to $0.6 \mathrm{ohm} \cdot \mathrm{cm}$ ) but metallic behaviour is not induced below $300 \mathrm{~K}$. The magnetic moment on $\mathrm{Cu}^{2+}$ remains low but our studies did not show any antiferromagnetic ordering below $300 \mathrm{~K}$ in any of the Li-doped phases.
\end{abstract}

Keywords. Layered copper compounds; Li doping; high temperature superconductors.

\section{Introduction}

The compound, $\mathrm{Ca}_{0.85} \mathrm{Sr}_{0.15} \mathrm{CuO}_{2}$ with the layer structure (Siegrist et al 1988) is interesting for the following reasons: (i) It is the prototype of the high $T_{c}$ copper oxide superconductors (HTSC) of the $\mathrm{Bi}$ and Tl families, viz. $\mathrm{Bi}_{2} \mathrm{Sr}_{2} \mathrm{Ca}_{n-1} \mathrm{Cu}_{n} \mathrm{O}_{2 n+4}$ and $\mathrm{Tl}_{m} \mathrm{Ba}_{2} \mathrm{Ca}_{n-1} \mathrm{Cu}_{n} \mathrm{O}_{2 n+2+m} m=1,2 ; n=1,2,3$ (Rao 1988a, b; Rao and Raveau 1989; Ramakrishnan and Rao 1989). The $\left(\mathrm{Ca}_{0.85} \mathrm{Sr}_{0.15}\right)$ plane does not contain any oxygens and the $\mathrm{CuO}_{2}$ planes are all well-defined (figure 1). (ii) It is an insulator (semiconductor) similar to the parent $\mathrm{HTSC}$ phases, viz. $\mathrm{Bi}_{2} \mathrm{Sr}_{2} \mathrm{YCu}_{2} \mathrm{O}_{8.5}$, $\mathrm{TlBa}_{2} \mathrm{YCu}_{2} \mathrm{O}_{7}$ and $\mathrm{YBa}_{2} \mathrm{Cu}_{3} \mathrm{O}_{6}$ (Ganguli et al 1989; Hervieu et al 1988; Torardi et al 1987). (iii) The $\mathrm{Cu}^{2+}$ has a magnetic moment, despite a low value of $0.5 \mu \mathrm{B}$ and the compound orders antiferromagnetically with a fairly high Neel temperature $\left(T_{N}=537 \pm 5 \mathrm{~K}\right)$ (Vaknin et al 1989). This behaviour is analogous to the parent HTSC insulating phases which order antiferromagnetically at high temperature. Hence a detailed understanding of the properties of the prototype compound, $\mathrm{Ca}_{0.85} \mathrm{Sr}_{0.15} \mathrm{CuO}_{2}$, can help in elucidating the mechanism of $\mathrm{HTSC}$ in the mixed copper oxides. It is to be noted that pure $\mathrm{CaCuO}_{2}$ does not have a layer structure and incorporation of $\mathrm{Sr}$ at the $\mathrm{Ca}$-site is essential to stabilize the desired layer structure.

In the present work we have attempted to introduce charge carriers by doping with monovalent $\mathrm{Li}$ ions at the Ca-site. In this process mixed valence can be created at the $\mathrm{Cu}$ site $\left(\mathrm{Cu}^{2+} / \mathrm{Cu}^{3+}\right)$ and hopping of holes resulting in improved conductivity. In the ideal case, we may expect a metallic behaviour similar to $\mathrm{La}_{2}-\mathrm{Sr}_{x} \mathrm{CuO}_{4}$. We present the $\mathrm{X}$-ray, magnetic susceptibility and resistivity data for the phases, $\mathrm{Li}_{x}\left(\mathrm{Ca}_{0.85} \mathrm{Sr}_{0 \cdot 15}\right)_{1-x} \mathrm{CuO}_{2}(x=0-0 \cdot 15)$.

\section{Experimental}

Compounds were prepared in polycrystalline form by the high temperature solid-state reaction of high purity $\mathrm{CaCO}_{3}(\mathrm{Cerac}, 99.99 \%), \mathrm{SrCO}_{3}(\mathrm{Cerac}, 99.99 \%$ ), $\mathrm{CuO}$ (Cerac, 


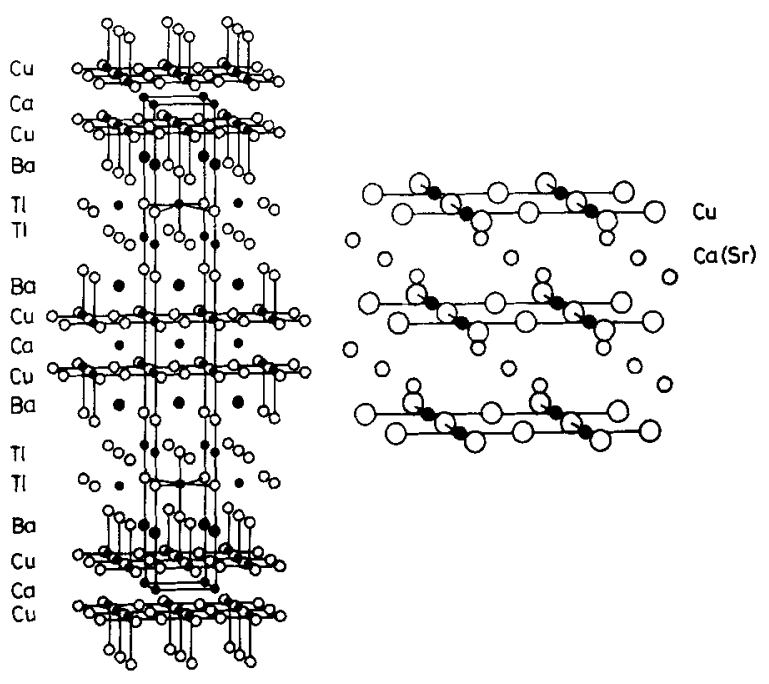

Figure 1. Structures of $\mathrm{Tl}_{2} \mathrm{Ba}_{2} \mathrm{Ca}_{1} \mathrm{Cu}_{2} \mathrm{O}_{8}$ and $\mathrm{Ca}_{0 \cdot 85} \mathrm{Sr}_{0 \cdot 15} \mathrm{CuO}_{2}$.

$99.9 \%$ ), $\mathrm{Li}_{2} \mathrm{CO}_{3}(\mathrm{Cerac}, 99.99 \%$ ) in stoichiometric amounts. The mixture was calcined at $910^{\circ} \mathrm{C}$ for $24 \mathrm{~h}$ and cooled, reground and heated at $920^{\circ} \mathrm{C}$ for $24 \mathrm{~h}$. The black powder was pelletized (WC-lined stainless steel die and plungers; $8 \mathrm{~mm}$ dia, $1-2 \mathrm{~mm}$ thick; 3 tons $/ \mathrm{cm}^{2}$ pressure) and again heated in air at $930^{\circ} \mathrm{C}$ for $24 \mathrm{~h}$ and cooled. The Li-doped samples were prepared in the range of $0 \leqslant x \leqslant 0 \cdot 25$ in steps of 0.05 .

The phase formation was tested by X-ray diffraction (XRD) using a Phillips, PW1140 unit ( $\mathrm{CuK} \alpha$-rad; Ni- filter; $40 \mathrm{kV} ; 20 \mathrm{~mA})$. Lattice parameters were obtained by LSQ fitting of the high angle $(h k l)$ lines. Electrical resistivity was measured on sintered pellets by four-probe Van der Pauw technique using a closed cycle He refrigerator (CTI cryogenics, USA; model $21 \mathrm{C}$ ) in the range $150-300 \mathrm{~K}$. Metallic indium contacts on the pellets were provided using an ultrasonic soldering iron. Magnetic susceptibility data were collected using the SQUID magnetometer (Quantum Design model 1822 MPMS, USA) in the range $4 \cdot 2-350 \mathrm{~K}$.

\section{Results and discussion}

\subsection{Structure and stability}

The compounds are black in colour and stable. The XRD patterns of the pure compound (figure 2) $\mathrm{Ca}_{0.85} \mathrm{Sr}_{0.15} \mathrm{CuO}_{2}$ indicated a single-phase formation and the lines could be indexed on the basis of tetragonal unit cell with space group $P 4 / \mathrm{mmm}$. The LSQ fitted lattice parameters given in table 1 are in very good agreement with those reported by Siegrist et al (1988). The XRD patterns of $\mathrm{Li}_{x}\left(\mathrm{Ca}_{0.85} \mathrm{Sr}_{0 \cdot 15}\right)_{1-x} \mathrm{CuO}_{2}$ are similar to that of the pure phase up to $x=0.15$ indicating single phase nature (figure 2 and table 1). Li doping decreases the $a$ and $c$ values. For $0 \cdot 2 \leqslant x \leqslant 0 \cdot 25$ impurity lines of $\mathrm{CuO}$ and $\mathrm{Ca}_{2} \mathrm{CuO}_{3}$ were seen in addition to those of 


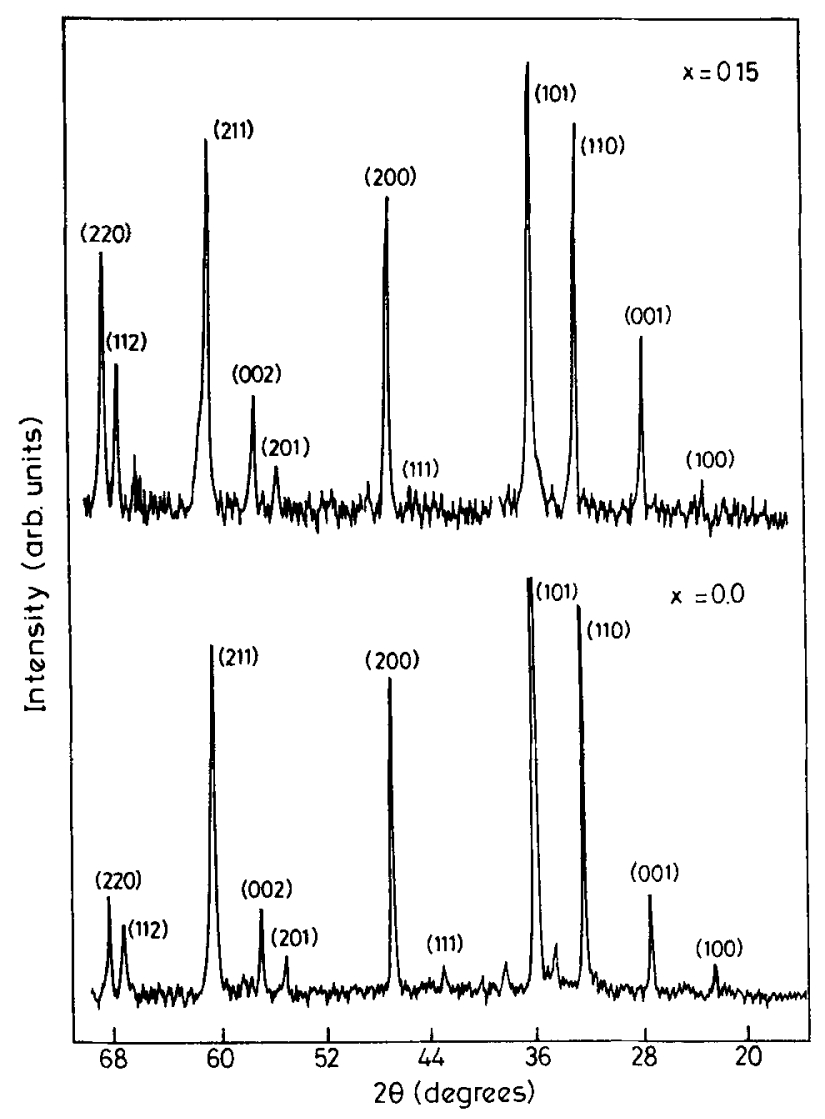

Figure 2. X-ray diffraction patterns of $\mathrm{Li}_{x}\left(\mathrm{Ca}_{0.85} \mathrm{Sr}_{0 \cdot 15}\right)_{1-x} \mathrm{CuO}_{2} . x$ values are shown.

Table 1. Structure and property data on the $\mathrm{Li}_{x}\left(\mathrm{Ca}_{0.85} \mathrm{Sr}_{0.15}\right)_{1-x} \mathrm{CuO}_{2}$ system

\begin{tabular}{|c|c|c|c|c|c|c|}
\hline \multirow[b]{2}{*}{ Composition } & \multicolumn{2}{|c|}{$\begin{array}{l}\text { Tetragonal } \\
\text { lattice param. }\end{array}$} & \multicolumn{2}{|c|}{ Resistivity, $\Omega \mathrm{cm}$} & \multirow[b]{2}{*}{$E_{a}(\mathrm{eV})$} & \multirow[b]{2}{*}{$p_{\text {eff }}$ B.M } \\
\hline & $a(\AA)$ & $c(\AA)$ & $\begin{array}{l}\text { Kesist } \\
\rho_{300 \mathrm{~K}}\end{array}$ & $\rho_{150 \mathrm{~K}}$ & & \\
\hline$x=0$ & $\begin{array}{l}3.88 \\
(3 \cdot 86)^{*}\end{array}$ & $\begin{array}{l}3.23 \\
(3.20)^{*}\end{array}$ & $3 \times 10^{3}$ & - & - & $\begin{array}{c}0 \cdot 1 \\
(0 \cdot 5)^{*}\end{array}$ \\
\hline$x=0.05$ & 3.88 & $3 \cdot 23$ & $2 \cdot 15$ & $25 \cdot 18$ & $\begin{array}{l}0.1(300-210 \mathrm{~K}) \\
0.06(210-150 \mathrm{~K})\end{array}$ & $0 \cdot 15$ \\
\hline$x=0.1$ & 3.87 & 3.22 & 0.78 & $15 \cdot 37$ & $0.08(300-150 \mathrm{~K})$ & $0 \cdot 21$ \\
\hline$x=0 \cdot 15$ & 3.87 & 3.22 & 0.55 & $21 \cdot 60$ & $0 \cdot 1(300-150 \mathrm{~K})$ & $0 \cdot 15$ \\
\hline
\end{tabular}

*Values in parenthesis are from Siegrist et al (1988) and Vaknin et al (1989).

$\mathrm{Ca}_{0.85} \mathrm{Sr}_{0 \cdot 15} \mathrm{CuO}_{2}$. For $x>0 \cdot 25$ the XRD patterns are complex and could not be indexed.

Substitution at the calcium site by $\mathrm{Mg}^{2+}, \mathrm{Ba}^{2+}, \mathrm{Y}^{3+}, \mathrm{La}^{3+}$ and $\mathrm{Ce}^{4+}$, at the copper site by $\mathrm{Fe}^{2+}$ and $\mathrm{Ni}^{2+}$, and at the oxygen site by $\mathrm{F}^{-}$was tried by the solid-state reaction method but did not yield single-phase materials. 


\subsection{Electrical properties}

The pure phase $\mathrm{Ca}_{0.85} \mathrm{Sr}_{0 \cdot 15} \mathrm{CuO}_{2}$ is found to be an insulator with a room temperature resistivity $\left(\rho_{300 \mathrm{~K}}\right) \sim 10^{3} \Omega \mathrm{cm}$ (table 1 ) as observed by Siegrist et al. As expected resistivity decreases with increasing Li content (table 1). Drastic decrease occurs for $x$ as small as 0.05 . Much smaller decrease in $\rho_{300 \mathrm{~K}}$ occurs for further increase in $x$. However, all compounds showed only semiconducting behaviour below room temperature and no metallicity was noted (figure 3 ). Measurements were restricted to the range of $150-300 \mathrm{~K}$ due to the high resistivity of the samples below $150 \mathrm{~K}$. No measurements could be carried out on the pure $\mathrm{Ca}_{0.85} \mathrm{Sr}_{0.15} \mathrm{CuO}_{2}$ phase below room temperature again due to high resistance.

The activation energy $\left(E_{a}\right)$ was calculated from the slope of the Arrhenius plot of $\log \sigma$ vs $1000 / T$ where $\sigma=1 / \rho \mathrm{ohm}^{-1} \mathrm{~cm}^{-1}$. The $E_{a}$ values are low $(0.06-0 \cdot 1 \mathrm{eV})$ for the Li-containing phases (table 1). For the $x=0.05$ sample we notice a break in the $\log \sigma$ vs $1 / T$ plot at $210 \mathrm{~K}$, the significance of which is not clear to us at present.

\subsection{Susceptibility measurements}

The molar susceptibility vs temperature plots for the pure and lithium-doped phases are shown in figure 4. Our value of $\chi_{M}$ for pure compound, viz. $1.3 \times 10^{-5} \mathrm{emu} / \mathrm{mole}$ at $300 \mathrm{~K}$ is smaller than the value of $4 \times 10^{-5} \mathrm{emu} / \mathrm{mole}$ reported by Vaknin et al (1989). However, the $\chi$-T dependence in the range of $50-300 \mathrm{~K}$ is the same in both measurements. The rise in $\chi_{M}$ below $50 \mathrm{~K}$ is due to the small amounts of magnetic impurities. For Li-doped compounds, the $\chi_{M, 300 \mathrm{~K}}$ values are large by an order of magnitude compared to the pure sample. The $\chi-T$ dependence in the range $140-300 \mathrm{~K}$ is more or less temperature-independent and $\chi_{M}$ increases below $140 \mathrm{~K}$. The data for

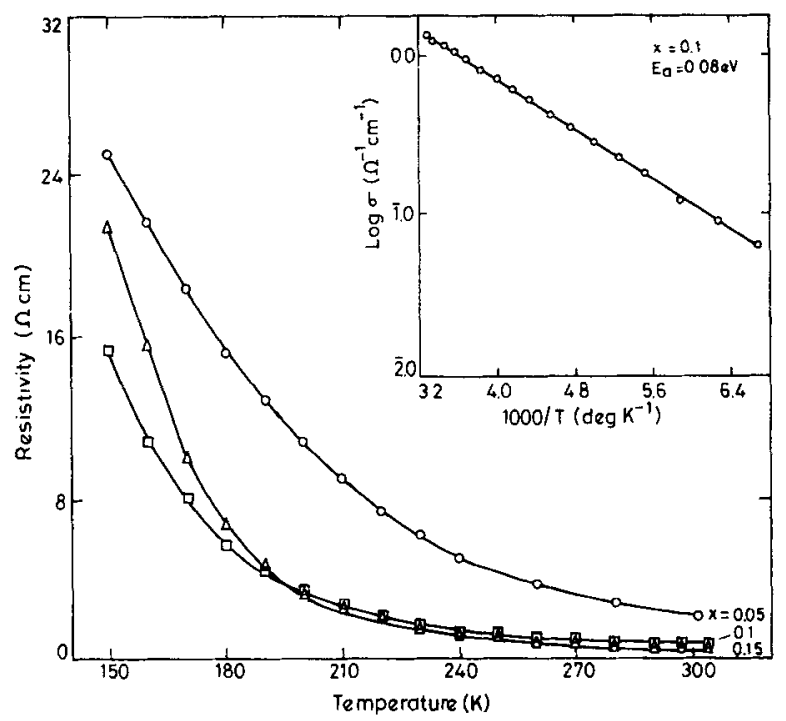

Figure 3. Resistivity plots of $\mathrm{Li}_{x}\left(\mathrm{Ca}_{0.85} \mathrm{Sr}_{0 \cdot 15}\right)_{1-x} \mathrm{CuO}_{2} . x$ values are shown. Inset is the Arrhenius plot to get activation energy. 


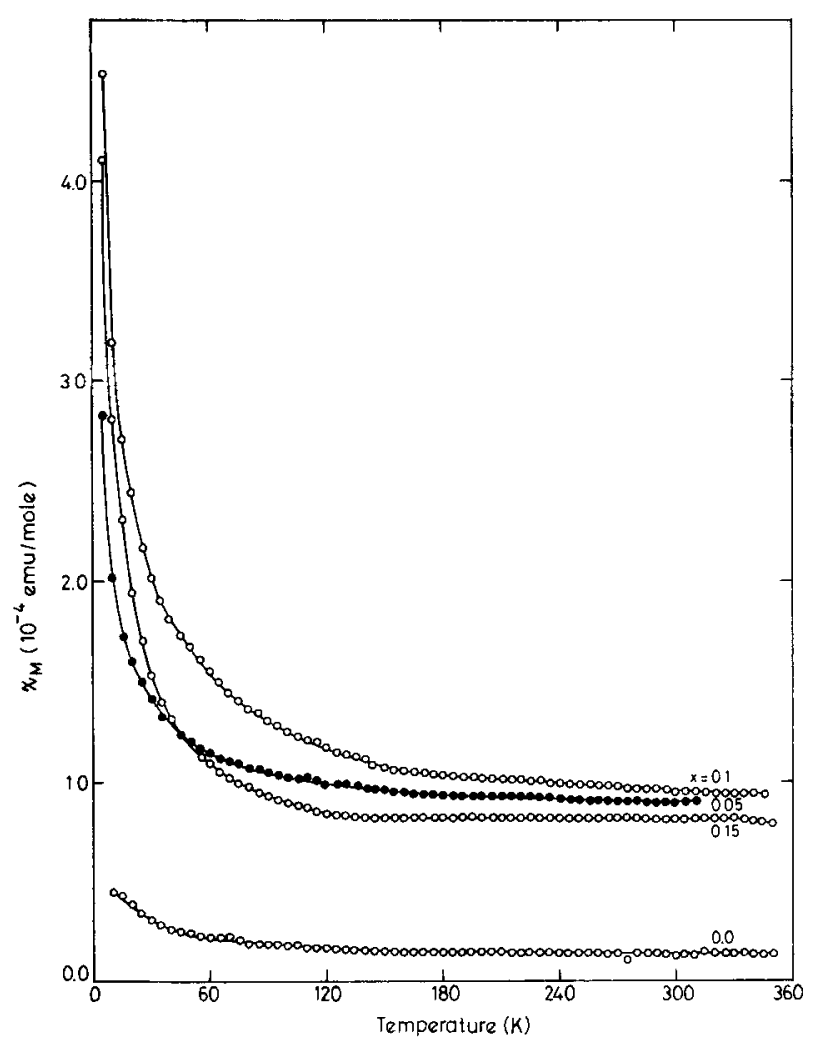

Figure 4. Magnetic susceptibility vs temperature plots for $\mathrm{Li}_{x}\left(\mathrm{Ca}_{0 \cdot 85} \mathrm{Sr}_{0 \cdot 15}\right)_{1-x} \mathrm{CuO}_{2}, x$ values are shown.

the pure and Li-doped $(x=0 \cdot 05,0 \cdot 1)$ phases were fit to a modified Curie Weiss law: $\chi=\chi_{0}+C /(T-\theta)$ where $\chi_{0}$ is temperature-independent susceptibility (a sum of core diamagnetic and Van Vleck contribution), $C$ the Curie constant and $\theta$ the Curie-Weiss temperature.

The effective magnetic moment $p_{\text {eff }}$ was calculated from the molar $C$ using the equation $p_{\text {eff }}=2 \cdot 83 C^{1 / 2}$. The values of $p_{\text {eff }}$ are listed in table 1 . The value for the pure phase is $0.1 \mu_{B}$. This is smaller than the value of $0.51 \pm 0.05 \mu_{B} / \mathrm{Cu}$ ion reported by Vaknin et al (1989) from their neutron diffraction data. We find that the $p_{\text {eff }}$ slightly increases for Li-doped compounds (table 1 and figure 4).

It is known that in the $\mathrm{La}_{2-x} \mathrm{Sr}_{x} \mathrm{CuO}_{4}$ system, with increase in $x$, the $\rho_{300 \mathrm{~K}}$ decreases due to the introduction of $\mathrm{Cu}^{3+}$ ions, and a change from semiconductor-to-metallic behaviour occurs for $x>0.05$ and superconductivity for $x \geqslant 0 \cdot 15$. Simultaneously, the antiferromagnetic $T_{N}$ decreases with increasing $x$. In the present case of $\mathrm{Li}_{x}\left(\mathrm{Ca}_{0.85} \mathrm{Sr}_{0.15}\right)_{1-x} \mathrm{CuO}_{2}$, we expect a decrease in $\rho_{300 \mathrm{~K}}$ with increase in $x$ as is indeed observed. However, no metallicity is induced. We also expect a decrease in $T_{N}$ with increase in $x$. However, we do not see any magnetic ordering, within the limits of our measurement, in the doped samples in the range $4 \cdot 2-300 \mathrm{~K}$. More careful studies including heat capacity data may throw light on this aspect. 


\section{Conclusions}

Present studies show that the tetragonal layer type compound $\mathrm{Ca}_{0.85} \mathrm{Sr}_{0 \cdot 15} \mathrm{CuO}_{2}$ is a semiconductor with $\rho_{300 \mathrm{~K}} \approx 10^{3} \Omega \mathrm{cm}$ and is paramagnetic with $p_{\text {eff }}=0 \cdot 1 \mu_{B}$ due to the highly correlated nature of the $\mathrm{Cu}^{2+}-3 d$ spins. Lithium can be doped to the extent of 0.15 and produces a drastic decrease in the resistivity of the compound. However, no metallic behaviour is noted below $300 \mathrm{~K}$. The $p_{\text {eff }}$ remains small in the Li-doped phases but no evidence for antiferromagnetic ordering is seen below $300 \mathrm{~K}$ in any of the doped phases.

\section{Acknowledgements}

Thanks are due to Prof. R Srinivasan of the Low Temperature Laboratory, Physics Department, for helpful discussions. Thanks are due to the Programme Management Board on Superconductivity and DST, New Delhi for providing research grant.

\section{References}

Ganguli A K, Nagarajan R, Nanjundaswamy K S and Rao C N R 1989 Mater. Res. Bull. 24103 Hervieu M, Maignan A, Martin C, Michel C, Provost J and Raveau B 1988 J. Solid State Chem. 75212 Ramakrishnan T V and Rao C N R 1989 J. Phys. Chem. 934414

Rao C N R (ed.) 1988a in Chemistry of oxide superconductors (Oxford: Blackwell Sci. Publ.)

Rao C N R (ed.) 1988b in Chemical and structural aspects of high temperature superconductors, (Singapore: World Scientific)

Rao C N R and Raveau B 1989 Acc. Chem. Res. 22106

Siegrist T, Zahurak S M, Murphy D W and Roth R S 1988 Nature (London) 334231

Torardi C C, Mc Carron E M, Bierstedt P E and Sleight A W 1987 Solid State Commun. 64497

Vaknin D, Caignol E, Davies P K, Fischer J E, Johnston D C and Goshorn D P 1989 Phys. Rev. B39 9122 